\section{- OPEN ACCESS}

\title{
Failure to improve door-to-needle time by switching to emergency physician-initiated thrombolysis for ST elevation myocardial infarction
}

\author{
Alexander Loch, ${ }^{1}$ Tint Lwin, ${ }^{2}$ Idzwan Mohd Zakaria, ${ }^{2}$ Imran Zainal Abidin, ${ }^{3}$ \\ Wan Azman Wan Ahmad, ${ }^{3}$ Oliver Hautmann ${ }^{4}$
}

- Additional material is published online only. To view please visit the journal online (http://dx.doi.org/10.1136/ postgradmedj-2012-131174).

${ }^{1}$ Department of Emergency Medicine/Cardiology, University Malaya Medical Centre, Kuala Lumpur, Malaysia

${ }^{2}$ Department of Emergency Medicine, University Malaya Medical Centre, Kuala Lumpur, Malaysia

${ }^{3}$ Department of Cardiology, University Malaya Medical Centre, Kuala Lumpur, Malaysia

${ }^{4}$ Department of Emergency Medicine/Trauma surgery, University Malaya Medical Centre, Kuala Lumpur, Malaysia

\section{Correspondence to}

Dr Alexander Loch,

Department of Emergency

Medicine/Cardiology, Pusat

Perubatan Universiti Malaya,

Lembah Pantai, Kuala Lumpur

59100, Malaysia;

alexanderloch@gmx.de

Received 30 May 2012 Revised 11 February 2013 Accepted 3 March 2013 Published Online First 22 March 2013

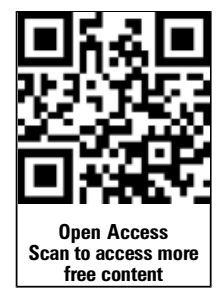

To cite: Loch A, Lwin T, Zakaria IM, et al. Postgrad Med J 2013;89:335-339.
ABSTRACT

Introduction Achieving target door-needle times for ST elevation myocardial infarction remains challenging. Data on emergency department (ED) doctor-led thrombolysis in developing countries and factors causing delay are limited.

Objectives To assess the effect on door-needle times by transferring responsibility for thrombolysis to the ED doctors and to identify predictors of prolonged door-needle times.

Methodology Data on medical on-call team-led thrombolysis at a tertiary Asian hospital were prospectively collected from May 2007 to Aug 2008 (1st study period). In September 2008, ED doctors were empowered to perform thrombolysis. The practice change was accompanied by new guidelines, tick chart implementation, and training sessions. Data were then consecutively collected from September 2008 to May 2009 (2nd study period). Door-to-needle times for the 1st and 2nd study periods were compared. All cases were analysed for factors of delay by multiple logistic regression.

Results 297 patients were thrombolysed, 169 by the medical on-call team during the 1st study period and 128 by the ED doctors during the 2 nd study period. Median door-needle times were 54 and $48 \mathrm{~min}$, respectively $(p=0.76)$. Significant delays were predicted by 'incorrect initial ECG interpretation' (adjusted OR (aOR) 14.3), 'inappropriate triage' (aOR 10.4) and 'multiple referrals' (aOR 5.9). No cases of inappropriate thrombolysis were recorded.

Conclusions Transfer of responsibility for thrombolysis to the ED doctors did not improve door-needle times despite measures introduced to facilitate this change. Key causative factors for this failure were identified.

\section{INTRODUCTION}

Primary percutaneous coronary intervention (PCI) is the preferred reperfusion strategy for ST elevation myocardial infarction (STEMI) provided it can be performed in a timely manner at a facility with a high level of experience. In our setting, like in most developing countries, primary PCI cannot be routinely provided round-the-clock because of logistical, economic and political issues. Furthermore, the delays very often associated with transferring a patient to the cardiac catheterisation laboratory might negate the potential benefit of primary $\mathrm{PCI}$ over immediate thrombolysis. Thrombolysis for acute STEMI remains a major treatment modality in developing countries, but also in established healthcare systems. ${ }^{1}$
Reported door-needle times in neighbouring countries range from $210 \mathrm{~min}$ in Bangladeshi hospitals $^{2}$ to $19 \mathrm{~min}$ in urban Japan. ${ }^{3}$ Established western healthcare systems also struggle to meet performance targets: a recent study in 178 American hospitals established that only $44.5 \%$ of patients were thrombolysed within the target time ${ }^{4}$-data confirming the general difficulties in achieving thrombolysis goals.

The benefit of any reperfusion therapy is strongly time dependent. Delay in reperfusion therapy is associated with increased in-hospital mortality. ${ }^{5}$ Relative reductions in mortality of up to $50 \%$ have been observed when therapy was provided early. ${ }^{6}$ Every attempt should be made to initiate thrombolysis early. Standardised protocols to ensure prompt reperfusion are recommended; however, evidence on the efficiency of such protocols is scarce. Factors identified as leading to delays in the administration of thrombolysis include 'delay in decision making' and 'inappropriate low-acuity triage' among others. ${ }^{7}$ Systematic studies into the whole spectrum and magnitude of such specific factors are lacking.

At our centre, thrombolytic therapy was traditionally administered by the medical on-call team. Previous audits highlighted the fact that doorneedle time targets were not met. In an attempt to improve door-needle times, an intervention was introduced in September 2008 in which emergency department (ED) doctors were given the authority to initiate thrombolysis directly. The primary objective of this study was to prospectively assess the effect on door-needle times of changing the responsibility for thrombolysis from the medical on-call team to the ED doctors. The secondary objective was to identify factors predicting door-to-needle times beyond the stipulated target of $30 \mathrm{~min}$.

\section{METHODOLOGY}

\section{Setting}

University Malaya Medical Centre is a 1279-bed tertiary urban hospital with more than 120000 annual ED attendances. Although primary and rescue angioplasty can be provided under limited circumstances, thrombolysis remains the main treatment for STEMI. Until September 2008, the clinical pathway for most patients presenting to the ED with acute myocardial infarction was as follows. The ED doctors were responsible for work up and diagnosis. The patients were subsequently referred to the medical officer to confirm the diagnosis and to 
decide about thrombolysis. The ED doctors were mostly junior trainees in Masters of Emergency Medicine (the UK equivalent of the specialist training programme). The medical officer was the equivalent of an SHO (senior house officer) in general medicine.

\section{Study intervention}

In an attempt to improve door-needle times, ED doctors were given the authority to initiate thrombolysis directly starting in September 2008. Two time periods were analysed for this study: the 1st study period included all patients thrombolysed by the medical officers from 1 May 2007 until 31 August 2008. The 2nd study period included all patients thrombolysed by the ED doctors from 1 September 2008 until 31 May 2009. All data were prospectively collected (see online supplementary box S1).

A set of guidelines for STEMI management was developed to help the ED doctors during the transition. The guidelines defined situations when ED doctor-initiated thrombolysis was appropriate and provided information on diagnostic criteria for thrombolysis and a list of contraindications and complications. Doctors were trained to complete a tick box chart guiding them through the process of decision making and thrombolysis administration (see online supplementary appendix 1). All ED doctors had to complete a training module consisting of lectures before the study. Lectures were repeated at 6-monthly intervals. Wall posters detailing STEMI management were hung up in the department.

\section{Predictors of door-to-needle times of more than $30 \mathrm{~min}$}

A small pilot study was conducted before the actual study to identify factors potentially explaining prolonged door-to-needle times. Records of 50 patients with significantly delayed thrombolysis administration were retrospectively reviewed by an emergency medicine specialist and a cardiologist for possible causes. The factors identified included: 'multiple referrals' (defined as three or more doctors involved in the decision-making process); 'inappropriate triage' (defined as inappropriately triaging a patient to a lower priority zone on arrival); 'incorrect initial ECG interpretation' (defined as failure to interpret the initial ECG as STEMI despite the presence of ST elevation as assessed by a senior cardiologist); 'need for resuscitation before thrombolysis'; 'evolving STEMI' (defined as STEMI criteria not met on the initial ECG with development of STEMI ECG criteria while in the ED); 'delayed drug preparation' (defined as $>15 \mathrm{~min}$ needed for the nursing staff to prepare and start the infusion after being instructed by the doctors to do so); 'need for prior investigations' (eg, to rule out aortic dissection or acute stroke); 'unusual presentations' (defined as presentation with complaints other than chest pain and an incidental finding of STEMI on ECG). All cases in the study under discussion (regardless of whether or not the door-to-needle time target of $30 \mathrm{~min}$ was met) were reviewed again by an emergency specialist and a cardiologist for the presence or absence of the previously identified factors.

\section{Inclusion and exclusion criteria}

Patients fulfilling the universal definition of STEMI ${ }^{8}$ upon presentation and who received thrombolysis were included. Patients with the need for investigations to exclude conditions such as pulmonary embolism or dissecting aneurysm and patients requiring cardiopulmonary resuscitation or treatment of uncontrolled hypertension before thrombolysis were included in the study. Patients referred from other hospitals, patients declining thrombolysis or undergoing primary PCI were excluded. The only available thrombolytic agent at our centre is streptokinase, which is administered at a dose of 1.5 million units by intravenous infusion over $60 \mathrm{~min}$.

\section{Data collected and statistical analysis}

Data collection included age, sex, race, infarct location, complications and survival to discharge or death. Time of onset of chest pain, time of arrival in the ED, and time of the start of thrombolytic therapy were recorded. 'Door time' is the registration time in the ED. 'Door-needle time' is the time interval in minutes between registration and initiation of thrombolysis. Symptom-to-door and door-to-needle times were calculated. Complications arising from the thrombolysis were recorded. A team consisting of an emergency medicine specialist and a cardiologist assessed appropriateness of thrombolysis by reviewing all cases. Inappropriate thrombolysis was defined as the provision of thrombolysis to patients with a final diagnosis other than STEMI, non-adherence to thrombolysis criteria, or thrombolysis for late presenters.

Failure of thrombolysis was defined as less than a 50\% reduction in ST elevation height in the worst infarcted lead after $1 \mathrm{~h}$, ongoing chest pain despite completed thrombolysis, or development of cardiovascular instability. Patients with failed thrombolysis underwent rescue PCI.

Statistical analysis was performed using SPSS V.14. The differences between door-needle times between the 1st and 2 nd study period were analysed statistically using an independent $t$ test The factors contributing to delay in the $\leq 30 \mathrm{~min}$ group and $>30$ min group were statistically analysed using $\chi^{2}$ followed by multiple logistic regression in order to identify significant predictors of prolonged door-to-needle times. Statistical significance was ascribed at $\mathrm{p}$ values $<0.05$.

\section{RESULTS}

A total of 297 patients with STEMI were thrombolysed during the study period: 169 were thrombolysed by the medical on-call team (1st study period; from 1 May 2007 until 31 August 2008), and 128 were thrombolysed by the ED doctors after the intervention (2nd study period; from 1 September 2008 until 31 May 2009). All patients were loaded with aspirin $300 \mathrm{mg}$ and clopidogrel $300 \mathrm{mg}$.

\section{Patients}

The mean \pm SD age was $54 \pm 12$ (range $25-86$ ) years. A total of 264 male $(88.9 \%)$ and 33 female $(11.1 \%)$ patients participated. There was no difference between the groups with regard to baseline characteristics (table 1).

\section{Door-to-needle time}

The mean \pm SD door-to-needle time was 69 \pm 59 (range 4-447) min with a median of 54 min during the 1 st study period and $71 \pm 61$ (range 2-330) min with a median of 48 min during the 2nd study period (after implementation). There was no significant difference between the groups (table 2). There was no difference in the mean door-needle time for patients presenting during office hours (08.00 h-17.00 h, Mondays to Fridays) or non-office hours (72 $\mathrm{min}$ and $71 \mathrm{~min}$, respectively; $\mathrm{p}=0.95$ ).

\section{Factors predicting prolonged door-needle times}

The prevalence of these factors in all patients is shown in figure 1. The presence of these factors was analysed in all cases independently of whether or not the recommended door-needle time was achieved. Factors shown on binary logistic regression to predict significantly prolonged door-to-needle times are incorrect initial ECG interpretation (adjusted OR (aOR) 14.3 (95\% CI 1.9 to 
Table 1 Baseline characteristics of study population

\begin{tabular}{|c|c|c|c|}
\hline & $\begin{array}{l}\text { 1st study period } \\
(n=169)\end{array}$ & $\begin{array}{l}\text { 2nd study period } \\
(n=128)\end{array}$ & $\begin{array}{l}p \\
\text { Value }\end{array}$ \\
\hline \multicolumn{4}{|l|}{ Demography } \\
\hline Mean age & 55 years & 55 years & 0.48 \\
\hline Male:female & $145: 24$ & $118: 10$ & \\
\hline \multicolumn{4}{|l|}{ Infarct area } \\
\hline Anterior & 94 & 50 & 0.10 \\
\hline Inferior & 68 & 69 & \\
\hline Lateral & 4 & 3 & \\
\hline Posterior & 1 & 2 & \\
\hline \multicolumn{4}{|l|}{ Comorbidities } \\
\hline Hypertension & 86 & 53 & 0.13 \\
\hline Diabetes & 66 & 47 & 0.76 \\
\hline Smoking & 85 & 66 & 0.83 \\
\hline
\end{tabular}

110.9)), inappropriate triage (aOR 10.4 (95\% CI 1.3 to 82.6)) and multiple referrals (aOR 5.9 (95\% CI 3.2 to 10.9)) (table 3 ).

\section{Complications and outcome}

There were no cases of inappropriate thrombolysis. Thrombolysis failure occurred in 61 (20.5\%) patients.

\section{DISCUSSION}

From this study, there was failure to improve door-needle times by handing the responsibility to initiate thrombolysis to the ED doctors despite measures introduced to facilitate this change (formal training sessions, guidelines, checklists). Our secondary objective identified the significant predictors of delay in initiating thrombolytic therapy as incorrect ECG interpretation (aOR 14.3), followed by inappropriate triage (aOR 10.4) and multiple referrals (aOR 5.9).

Our intervention aimed mainly to improve door-needle times by facilitating the correct diagnosis of STEMI in a timely manner and by shortening the referral chain. Unfortunately, we were unable to do so for several reasons. In our setting, where the number of specialists is very limited, most of the patients are initially seen by junior doctors, with experience in the field of emergency medicine ranging from only 1 to 3 years. During the study period, cover by consultants in emergency medicine was only available during office hours. In many cases, instead of thrombolysing directly when it was appropriate, the ED doctors referred the cases to the medical officer, who then often referred the case to a junior cardiologist. Many cases involved a senior cardiologist making the final decision. Therefore, we hypothesise that lack of experience paired with fear of thrombolysing patients led to both a high rate of incorrect ECG interpretation and an increased referral rate, resulting in duplicate consultations before arrival at a

Table 2 Door-needle times

\begin{tabular}{lllll}
\hline & $\begin{array}{l}\text { Total } \\
(\mathbf{n}=297)\end{array}$ & $\begin{array}{l}\text { 1st study period } \\
(\mathbf{n}=169)\end{array}$ & $\begin{array}{l}\text { 2nd study period } \\
(\mathbf{n}=\mathbf{1 2 8})\end{array}$ & $\begin{array}{l}\mathbf{p} \\
\text { Values }\end{array}$ \\
\hline Mean & $69 \mathrm{~min}$ & $69 \mathrm{~min}$ & $71 \mathrm{~min}$ & $0.759^{*}$ \\
Median & $50 \mathrm{~min}$ & $54 \mathrm{~min}$ & $48 \mathrm{~min}$ & \\
$<30 \mathrm{~min}$ & $73(25 \%)$ & $42(25 \%)$ & $31(24 \%)$ & 0.931 \\
\hline \multicolumn{4}{l}{ *Independent samples t test. }
\end{tabular}

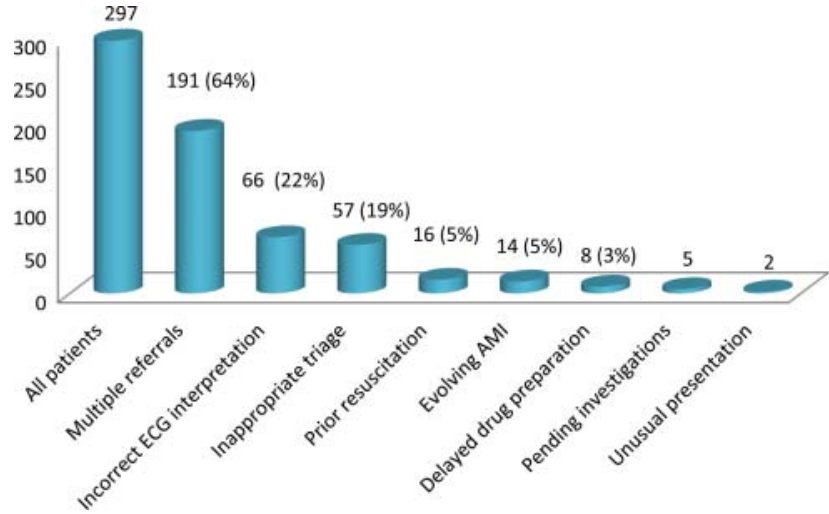

Figure 1 Factors leading to prolonged door-needle times. AMI, acute myocardial infarction. This figure is only reproduced in colour in the online version.

decision. It has been shown that a cardiologist review of patients before thrombolysis invariably results in an increase in the doorneedle times beyond the recommended $30 \mathrm{~min} .{ }^{9}$ This study highlights that effective decision making cannot be instilled by printed guidelines and lectures alone and that, in order to implement change, senior supervision for junior doctors is essential. This is in keeping with an Australian study where a multifaceted implementation strategy mainly consisting of teaching sessions on its own had no effect on door-needle times. ${ }^{10}$ Similarly, other studies have identified that one of the keys to success was adequate cover for the junior staff by experienced doctors. 911

The other significant factor for delay was inappropriate triage, and this was not addressed by the intervention, which implemented changes only after the patients had been triaged. This finding is supported by a Canadian study, where $50 \%$ of patients with STEMI in Canadian hospitals were inappropriately triaged to low-acuity areas resulting in significant delays. ${ }^{7}$ Our study findings are summarised in the main messages.

\section{Limitations}

Some patients admitted with STEMI during the study period might have been missed, as there are occasionally direct admissions to the cardiology team, bypassing the ED. The study was a prospective observational intervention study that monitored the effect of an intervention (change of practice) on a measurable clinical quality indicator (door-to-needle time) with its inherent limitations - that is, proneness to bias and lack of generalisability. At the time of the study, there was still a lack of qualified emergency medicine specialists, resulting in limited supervision and support of junior doctors. In an environment of better support, the results might have been different. The small study size might have resulted in the omission of other significant predictors of delay.

\section{Strategies to improve outcomes based on study findings and future research}

In view of the study findings, we aimed to improve door-needle times by specifically addressing the identified factors of delay. Triage courses for nurses have been set up to target inappropriate triage practices. All patients with chest pain are identified during primary triage and receive immediate attention and an ECG. A secondary triage for patients categorised during primary triage as low priority or 'non-cardiac' has been established. During secondary triage, ECGs are being recorded for all low-priority patients within $15 \mathrm{~min}$ of arrival resulting in an earlier identification of atypically presenting STEMIs. 
Table 3 Factors predicting prolonged door-to-needle times

\begin{tabular}{|c|c|c|c|c|c|c|}
\hline Factor & COR & $95 \% \mathrm{Cl}$ & $p$ Value & $\mathrm{aOR}$ & $95 \% \mathrm{Cl}$ & $\mathrm{p}$ Value \\
\hline Incorrect ECG interpretation & 30.0 & 4.1 to 220.7 & 0.001 & 14.3 & 1.9 to 110.9 & 0.011 \\
\hline Inappropriate triage & 24.5 & 3.3 to 180.2 & 0.001 & 10.4 & 1.3 to 82.6 & 0.027 \\
\hline Multiple referrals & 7.4 & 4.1 to 13.3 & 0.001 & 5.9 & 3.2 to 10.9 & 0.001 \\
\hline Need for resuscitation & 5.3 & 0.7 to 40.6 & 0.076 & 5.5 & 0.7 to 45.6 & 0.110 \\
\hline Evolving AMI & 4.5 & 0.6 to 35.2 & 0.115 & 3.8 & 0.5 to 31.6 & 0.220 \\
\hline Delayed drug preparation & 2.4 & 0.3 to 19.6 & 0.410 & 1.8 & 0.2 to 17.9 & 0.630 \\
\hline Awaiting investigations & 1.3 & 0.2 to 12.1 & 0.798 & 1.7 & 0.2 to 17.4 & 0.670 \\
\hline Unusual presentation & 0.3 & 0.02 to 5.3 & 0.410 & 0.2 & 0.01 to 15.9 & 0.180 \\
\hline
\end{tabular}

aOR, adjusted OR; cOR, crude OR; Ml, acute myocardial infarction.

ECG interpretation is given more emphasis during the emergency specialist training, both at the bedside and in formal teaching sessions. A mechanism to immediately electronically forward the ECG to the cardiologist in the case of diagnostic uncertainty was established. To address the problem of redundant referrals, a 'downstream' activation of cardiology services was created where the most senior on-call cardiologist will immediately review the patient when requested by the ED doctor, as opposed to multiple junior doctors referring up the chain of seniority. A $24 \mathrm{~h}$ cover with emergency specialists has been implemented.

Models advocating direct access to the Coronary Care Unit have been explored as a result of other studies showing impressive results, but may not be relevant in a setting such as ours where most patients self-present to the ED. ${ }^{12}{ }^{13}$ Nurse-initiated thrombolysis has also been shown to significantly reduce door-to-needle times, but such a model might not be feasible in our healthcare systems at the present time because of lack of trained nurses. ${ }^{14}$ Other potential areas for improvement that require further study are listed in box 1 .

\section{CONCLUSION}

Our intervention where the responsibility of thrombolysis was transferred to the ED doctors did not improve door-needle times despite measures introduced to facilitate this change (formal training sessions, guidelines, checklists). Thrombolysis performed by ED doctors in a developing Asian country was safe and achieved median door-needle times comparable to that of the medical on-call team. Predictors of delayed

\section{Box 1 Current research questions}

In the setting of a tertiary hospital in a megacity of a developing Asian country, the following research questions merit attention:

- Large-scale studies of prehospital thrombolytic therapy in congested Asian cities

- Impact of prehospital 12-lead ECG forwarding and telemedicine in ambulances on door-needle and door-balloon times

- Effect of increased number of emergency specialists on door-needle times

- Studies to look at the whole health system for care of patients with ST elevation myocardial infarction, including the ambulance response and interfaces between all the emergency services administration of thrombolysis included incorrect initial ECG interpretation, inappropriate triage and multiple referrals, which were not resolved by the intervention.

\section{Main messages}

- Thrombolysis performed by emergency department (ED) doctors in a developing Asian country was safe and achieved median door-to-needle times comparable to that of the medical on-call team.

- Transfer of responsibility for thrombolysis from the medical on-call team to ED doctors combined with measures to facilitate this change (formal training sessions, guidelines, checklists) did not, however, result in door-needle times within recommended targets ( $<30 \mathrm{~min}$ ).

- Interventions aimed at improving door-needle times should be preceded by a detailed factor analysis to target problem areas specifically.

- Key predictors of delayed thrombolysis are:

- multiple referrals

- incorrect triage of patients with ST elevation myocardial infarction

- incorrect initial ECG diagnosis by ED doctors

\section{Key references}

- Glickman SW, Cairns CB, Chen AY, et al. Delays in fibrinolysis as primary reperfusion therapy for acute ST-segment elevation myocardial infarction. Am Heart $J$ 2010;159:998-1004 e2.

- Irwani I, Seet CM, Manning PG. Emergency physician versus cardiologist-initiated thrombolysis for acute myocardial infarction: a Singapore experience. Singapore Med J 2004;45;313-17.

- Corfield AR, Graham CA, Adams JN, et al. Emergency department thrombolysis improves door to needle times. Emerg Med J 2004;21:676-80.

Contributors $\mathrm{AL}, \mathrm{TL}$ and IZA conceived and designed the study. AL, TL, IZA and IMZ developed the study protocol. $\mathrm{AL}, \mathrm{TL}, \mathrm{OH}$ and IMZ supervised data collection. $\mathrm{AL}$ and $\mathrm{TL}$ analysed the data. $\mathrm{AL}, \mathrm{TL}, \mathrm{OH}$ and WAWA prepared and approved the manuscript.

Competing interests None.

Ethics approval University Malaya ethics committee, Kuala Lumpur, Malaysia. 
Provenance and peer review Not commissioned; externally peer reviewed.

Open Access This is an Open Access article distributed in accordance with the Creative Commons Attribution Non Commercial (CC BY-NC 3.0) license, which permits others to distribute, remix, adapt, build upon this work non-commercially, and license their derivative works on different terms, provided the original work is properly cited and the use is non-commercial. See: http://creativecommons.org/ licenses/by-nc/3.0/

\section{REFERENCES}

1 McKiernan CJ, Taylor SG, Graham CA. Treatment of acute myocardial infarction in Scottish emergency departments: survey of current practice. Eur J Emerg Med 2006;13:43-5.

2 Muqueet MA, Haque KM Sirajul, Faruque GM, et al. An evaluation of door to needle time (DNT) of thrombolytic therapy following acute myocardial infarction in three large tertiary referral hospitals in Dhaka City. Bangladesh Med Res Counc Bull 2006:32:29-34.

3 Fukuoka Y, Dracup K, Ohno M, et al. Predictors of in-hospital delay to reperfusion in patients with acute myocardial infarction in Japan. J Emerg Med 2006;31:241-5.

4 Glickman SW, Cairns CB, Chen AY, et al. Delays in fibrinolysis as primary reperfusion therapy for acute ST-segment elevation myocardial infarction. Am Heart J 2010;159:998-1004 e2.
5 Rathore SS, Curtis JP, Chen J, et al. Association of door-to-balloon time and mortality in patients admitted to hospital with ST elevation myocardial infarction: national cohort study. BMJ 2009;338:b1807.

6 Boersma E, Maas AC, Deckers JW, et al. Early thrombolytic treatment in acute myocardial infarction: reappraisal of the golden hour. Lancet 1996:348:771-5.

7 Atzema CL, Austin PC, Tu JV, et al. Emergency department triage of acute myocardial infarction patients and the effect on outcomes. Ann Emerg Med 2009;53:736-45.

8 Thygesen K, Alpert JS, White HD. Universal definition of myocardial infarction. J Am Coll Cardiol 2007;50:2173-95.

9 Irwani I, Seet CM, Manning PG. Emergency physician versus cardiologist-initiated thrombolysis for acute myocardial infarction: a Singapore experience. Singapore Med J 2004:45:313-17.

10 Kinsman L, Tori K, Endacott R, et al. Guideline implementation fails to improve thrombolytic administration. Accid Emerg Nurs 2007;15:27-33.

11 Corfield AR, Graham CA, Adams JN, et al. Emergency department thrombolysis improves door to needle times. Emerg Med J. 2004;21:676-80.

12 Yaylali YT. Door-to-needle times in acute myocardial infarction. Asian Cardiovasc Thorac Ann 2010;18:122-6.

13 Yaylali YT, Susam I, Ates A, et al. Impact of a well-organized collaborative team approach on mortality in patients with ST-segment elevation myocardial infarction. Anadolu Kardiyol Derg 2010;10:508-13.

14 Wilmshurst P, Purchase A, Webb C, et al. Improving door to needle times with nurse initiated thrombolysis. Heart 2000;84:262-6. 